\title{
Experimental Biology 2013
}

\section{0-24 APRIL 2013}

Boston Convention and Exhibition Center

Boston, MA

Attendance: $>13,000$ registered

Experimental Biology is a 'superconference' of sorts, sponsored by six societies representing different aspects of biological science: American Association of Anatomists (AAA), American Physiological Society (APS), American Society for Biochemistry and Molecular Biology (ASBMB), American Society for Investigative Pathology (ASIP), American Society for Nutrition (ASN) and American Society for Pharmacology and Experimental Therapeutics (ASPET). Each society planned a program of scientific presentations for the conference, highlights of which are presented here.

ASN emphasized a session on the safety and efficacy of caloric restriction for lifespan extension. Research in animals suggests that caloric restriction is highly effective for extending both overall lifespan and the period of healthy, active life with minimal side effects. But clinical trials to determine whether this is a feasible, effective and safe intervention for humans have been insufficient, despite enormous public interest in healthy aging and the avoidance of premature death in older adults. John Speakman (University of Aberdeen, Scotland) presented an overview of current research on caloric restriction in animals including rodents and nonhuman primates, showing that the effects on lifespan are quite mixed. Other speakers addressed insights on caloric restriction from both naturally occurring human populations and clinical trials. As in animals, caloric restriction in humans has mixed effects.

ASBMB chose to focus on triple-negative breast cancer, a particularly recalcitrant disease for which there are few treatment options aside from traditional chemotherapy and surgery. Triplenegative breast cancer tumors are often aggressive and have high rates of recurrence and mortality. ASBMB's special program, "Benchside to Bedside: Identifying Novel Biomarkers to Better Manage Breast Disease," covered the basics of breast cancer, how new technologies have identified potential therapeutic targets for triple-negative breast cancer and why the disease disproportionately affects black women.

As the AAA celebrated its 125th anniversary, it looked to the past with a feature on visualizing dinosaurs with advanced three-dimensional imaging. Lawrence Witmer, a paleontologist at Ohio University (Athens), discussed the Visible Interactive Dinosaur project, which aims to restore dinosaur head anatomy by reconstructing soft-tissue systems in a three-dimensional digital environment. Witmer's group also recently published an article that

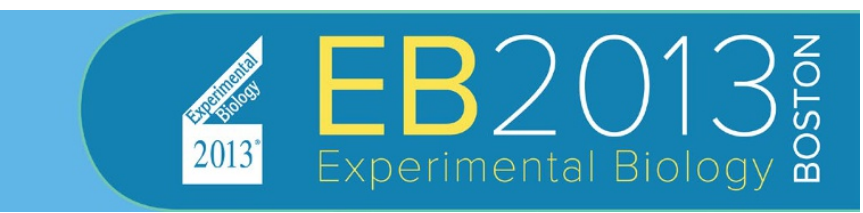

reports the use of these techniques to explore the feeding biology of Allosaurus (Paleontologica Electronica 16, 11A; 2013).

Meanwhile, ASIP emphasized the modern threat of emerging infections in a symposium that brought together scientists from the National Institutes of Health (Bethesda, MD) and the National Emerging Infectious Diseases Laboratory (Boston, MA). Katharine Bossart, Paul Duprex and Dorian McGavern highlighted new tools, treatments and vaccines and how they are changing the fight against emerging diseases such as henipavirus and morbillivirus.

APS targeted a different modern health problem in eating disorders: anorexia nervosa, bulimia nervosa and binge-eating disorder. Recent advances in investigative techniques, particularly the development of animal models of these disorders, have identified new mechanisms and treatment targets that may enhance our understanding and ability to develop successful treatments. Nicole Barbarich-Marsteller (Columbia University, New York, NY) and Mary Boggiano (University of Alabama, Birmingham) discussed translational results from rodent models of anorexia nervosa and binge-eating disorder.

Rounding out the conference program, ASPET presented the newest techniques in drug discovery and therapy, from 'organs-onchips' and three-dimensional 'bioprinting' of human tissues in vitro to microscale tissue engineering and the applications of silk as a biomaterial. These new technologies and research paradigms share the goal of increasing both the efficiency of the drug discovery process and the safety and efficacy of the resulting therapeutics.

In addition to the scientific sessions, each society provided career development sessions for the fields they represent, and APS, ASBMB and ASN presented public policy programs as well.

ASBMB teamed up with the Cambridge Science Festival for the "What is a Germ?" challenge, in which they solicited responses to that question framed in terms that grade-school students could understand. Submissions were accepted in any format (written, audio, pictorial, visual, etc.) and could be of any size or length. Students at Boston-area schools judged the submissions and chose the finalists, who presented their responses at the Cambridge Science Festival's Curiosity Challenge. The submissions are posted online at ASBMB's blog (http://theinteractome.wordpress.com/). The winning entry was a video titled "The Journey to the Germ" and submitted by the ASBMB's Undergraduate Affiliates Network chapter at Suffolk University (Boston, MA). Second place went to a rhyming, illustrated slide presentation by 11-year-old Simran Chandawarkar in which Oinky the pig learns why he is sick. 